\title{
Thermal Video Analysis for Fire Detection Using Shape Regularity and Intensity Saturation Features
}

\author{
Mario I. Chacon-Murguia and Francisco J. Perez-Vargas \\ Visual Perception Applications on Robotic Lab, Chihuahua Institute of Technology \\ mchaconaitchihuahua.edu.mx, fjperez@ieee.org
}

\begin{abstract}
This paper presents a method to detect fire regions in thermal videos that can be used for both outdoor and indoor environments. The proposed method works with static and moving cameras. The detection is achieved through a linear weighted classifier which is based on two features. The features are extracted from candidate regions by the following process; contrast enhance by the Local Intensities Operation and candidate region selection by thermal blob analysis. The features computed from these candidate regions are; region shape regularity, determined by Wavelet decomposition analysis and region intensity saturation. The method was tested with several thermal videos showing a performance of $4.99 \%$ of false positives in non-fire videos and $75.06 \%$ of correct detection with $7.27 \%$ of false positives in fire regions. Findings indicate an acceptable performance compared with other methods because this method unlike other works with moving camera videos.
\end{abstract}

Keywords: fire detection, thermal image processing, image segmentation.

\section{Introduction}

Fire detection is vital for early fire detection systems as well as in fire control. Fire detection systems may contribute to detect hazards situations that may reduce the danger for human lives as well as negative economic impact. Most of conventional fire detection systems are based on particle sampling techniques, temperature monitoring and air transparency. Unfortunately, these systems need to be located close to the fire and not always detect fire but smoke which not necessarily indicates fire. Conventional fire detectors used in buildings depend on the detection of smoke or fire particles [1], therefore they are not suitable for large areas. Besides, they cannot provide information of size, intensity or location of fire. These situations justify the research of fire detection based on vision systems which overcome the previous disadvantages of conventional methods. There are vision systems that work on the visible spectrum [1]-[4] analyzing color and movement but lack of robustness because of a high false positive rate due to colors similar to fire or illumination problems because of reflections. Also, conventional cameras cannot generate relevant images once dense smoke appears in the scene. Therefore, the proposed method described in this paper works with IR images acquired with a thermal camera. IR cameras have the advantage of generating relevant information even under smoke 
conditions and fire detection with low radiation in the visible spectrum generated by alcohol and hydrogen [5].

The contributions of the work reported in this paper are the following. The proposed method can be used as an indoor as well as an outdoor fire detector system. The camera does not need to be close to the fire. Besides, the proposed method considers typical flame characteristics like irregular contours and the peculiarity of being the dominant heat source in the scene. Irregular contour features are converted to contour distance vectors. Other characteristic used in the method is related to the capacity of the flames to generate a large amount of heat which in turn may produce saturation levels in the camera scale. These features can be computed in static and moving IR cameras which represents an important advantage with respect other methods based only on static cameras.

\section{Fire Detection Method}

A general description of the proposed fire detection method is the following. Prospective fire blob detection is achieved based on maximum temperature, [6]. Blob contrast enhancement is done using the technique described in [7]. Then a binarization threshold is computed and an area filtering is performed to define fire candidate blobs. The decision over those candidate blobs is finally made based on region shape regularity, determined by Wavelet decomposition analysis and region intensity saturation.

\subsection{Image Preprocessing}

The thermal images are processed as gray level images. Besides, in order to eliminate some information added by thermal images (date, scale, etc.) a ROI is defined on the original image. The next step is to enhance the contrast of the ROI by the Local Intensities Operation, LIO, in its intensity brightening operation (IBO) mode, [7]. With this method high gray level values (high temperature values) are enhanced. The IBO operator at coordinates $(x, y)$ is defined by

$$
G(x, y)=\prod_{k=0}^{8} z_{k} .
$$

where $z_{k}$ are the 8-neighbord pixels of the pixel $z_{0}$ located at $(x, y)$. Figure 1 shows examples before and after of the application of the IBO operator.

\subsection{Prospective Fire Blobs Location}

At this point, the image information is suitable to try to detect fire blob candidates. These blobs are found by determining the maximum gray level in the image, which in turn may correspond to high temperature areas

$$
\operatorname{gmax}=\{(x, y) \mid \max \{G(x, y)\}\} .
$$



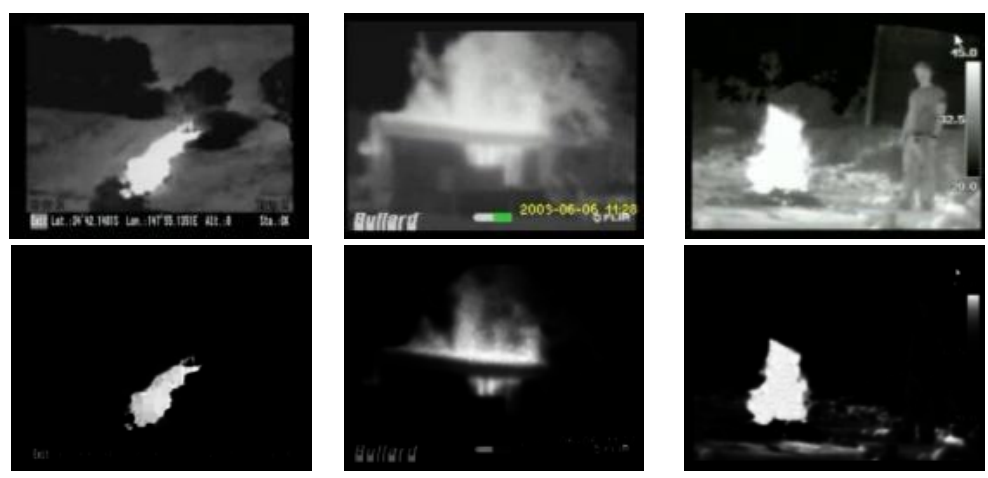

Fig. 1. Original images and fire blob enhanced after the IBO operator

Finding gmax does not necessary warranties that it corresponds to a fire blob. Therefore a minimum level for gmax needs to be determined. That is, a prospective fire blob must hold gmax $>\delta_{l}$. Considering non-fire as well as fire frames the value of $\delta_{l}$ was determined as 220 . If a prospective blob is located in the frame, the next step is to define the region of the fire blob. This area is defined as

$$
B(x, y)=\left\{\begin{array}{l}
1 \text { if } G(x, y)>\delta_{2} * \text { gmax } \\
0 \text { if } G(x, y)<\delta_{2} * \operatorname{gmax}
\end{array} .\right.
$$

where $\delta_{2}$ is a percentage threshold to define the pixels corresponding to the fire area. $\delta_{2}$ was defined to be a value between $70 \%$ and $85 \%$ based on experimentation.

The binary image $B(x, y)$ may contain noise regions, some false fire areas. In order to get rid of them an area filter is applied to $B(x, y)$

$$
F_{k}(x, y)=\left\{B_{k}(x, y) \mid \operatorname{Area}\left(B_{k}(x, y)\right)>40 \text { and } \operatorname{Area}\left(B_{k}(x, y)\right)>0.2 \alpha\right\} .
$$

where $F_{k}(x, y)$ is the $k$ prospective fire blob and $\alpha$ is the area of the largest region in $B(x, y)$. Figure 2 illustrates the process to determine prospective fire regions.

The previous thresholds and parameters were determined by statistical analysis using information of different videos taken with different cameras and conditions, therefore it is expected that the statistical validity is hold for other videos and cameras.

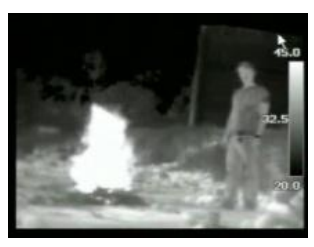

a)

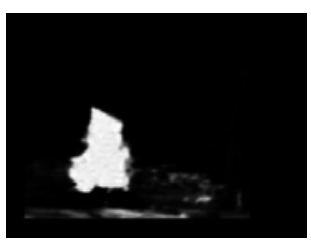

b)

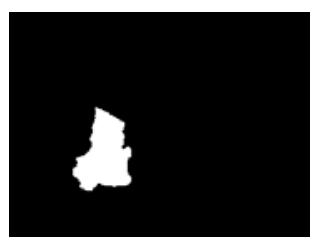

c)

Fig. 2. a) Original image, b) Pre-processed image, c) Candidate blob 


\subsection{Feature Extraction}

At this point the method has generated a set of fire candidate blobs. Therefore, it is necessary to design a classifier to determine if the prospective region $F_{k}(x, y)$ corresponds to a fire region. The features used in the classifier are related to the region shape regularity, determined by Wavelet decomposition analysis and region intensity saturation. The fire regions are mainly distinguishable from common objects or man-made objects as well as persons because fire regions present highly irregular contours, Figure 3 illustrates these cases. The irregularity analysis is performed in the Wavelet domain [8] as follows. A 1D signature $S[l]$ is obtained for $F_{k}(x, y)$ [9]. $S[l]$ contains the euclidean distance from the center of mass of $F_{k}(x, y)$ to its contour in function of the angle $\theta$ for $\theta=0$ to $360^{\circ}$. The Wavelet analysis is done according to the high and low pass filters proposed in [5],

$$
a[l]=s[l] * h[l] \text { and } d[l]=s[l] * g[l] \text {. }
$$

where

$$
h[l]=\left\{-\frac{1}{4}, \frac{1}{2},-\frac{1}{4}\right\} \text { and } g[l]=\left\{\frac{1}{4}, \frac{1}{2}, \frac{1}{4}\right\} .
$$

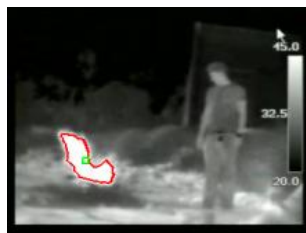

a)

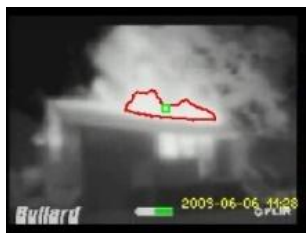

b)

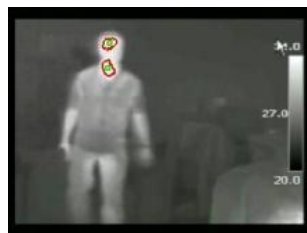

c)

Fig. 3. Center of mass and contour of candidate regions a) Bonfire, b) House on fire, c) Person

The Figure 4a shows the signatures as well as the Wavelet decomposition of candidate regions of Figure $3 \mathrm{a}$, fire region. On the other hand, Figure $4 \mathrm{~b}$ illustrates the case of a no-fire region of Figure 3c. It can be observed the differences of the signatures on the scales on both figures. This difference can be computed through an irregularity contour parameter $\beta$ expressed as,

$$
\beta=\sum_{l}|d[l]| / \sum_{l}|a[l]| .
$$

The irregularity parameter is normalized in order to be invariant to amplitude values. In this way, small values of $\beta$ correspond to non-fire regions. 


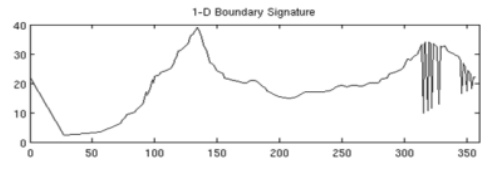

High Frecuency wavelet Coeficients
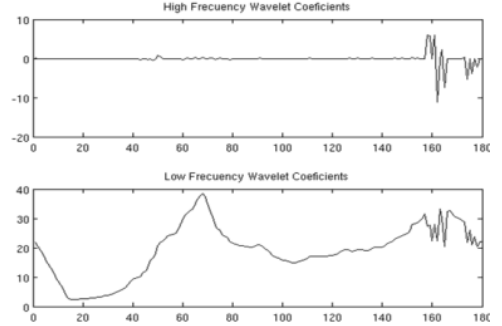

a)

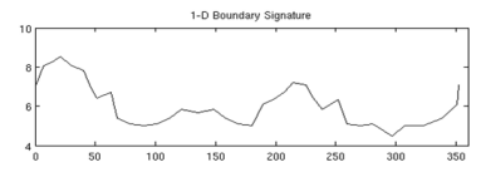

High Frecuency Wavelet Coeficients
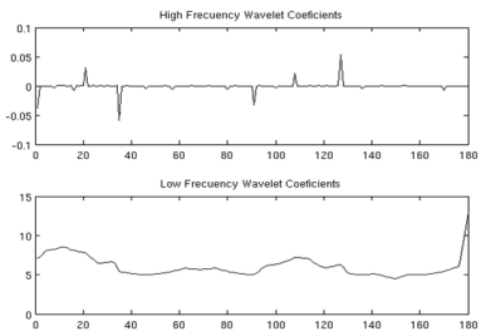

b)

Fig. 4. Signatures of candidate regions and their Wavelet decomposition of Figures, a) 3a,b) $3 c$

The second feature, intensity saturation, is related to the high temperature values associated to the fire. Since the fire region is the most prominent source of heat in the scene the pixel blob associated to it tends to reach the saturation level of the thermal camera [4].The intensity saturation feature is defined as

$$
\sigma=\pi / \tau
$$

where

$$
\begin{aligned}
& \pi=\left\|\left\{g(x, y) \mid(x, y) \in F_{K}(x, y)>\delta_{3}\right\}\right\| . \\
& \tau=\left\|\left\{g(x, y) \mid(x, y) \in F_{K}(x, y)\right\}\right\| .
\end{aligned}
$$

$g(x, y) \in G(x, y)$, II II stands for set cardinality. The threshold $\delta_{3}$ is computed automatically for each frame under analysis and must be close to the maximum level allowed for the radiometric resolution of the camera and in consequence greater than zero, that is

$$
\delta_{3}=\max \left\{\left.G(x, y)\right|_{(x, y) \in F_{k}(x, y)}\right\}-5 \text {. }
$$

Figure 5 illustrates the behavior of $\pi$ for a fire and a non-fire blob. As it was expected the intensity saturation level is greater in the fire region than in the non-fire blob, $\sigma=$ 0.9125 and $\sigma=0.3072$ respectively.

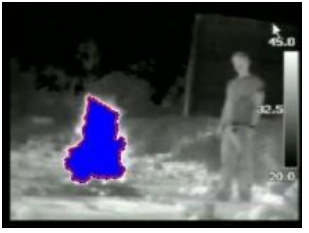

a)

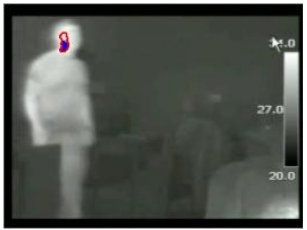

b)

Fig. 5. Illustration of saturation in a) Fire, b) Non-fire 


\subsection{Classification Scheme}

As a first approach and in order to keep the computational cost low a linear classifier was chosen. Future work will include analysis with other classifiers. The classification of the candidate regions is determined by the following rule

$$
F_{k}(x, y) \text { is fire if } \gamma>0.275 \text {. }
$$

where

$$
\gamma=w_{1} \beta+w_{2} \sigma .
$$

$w_{l}$ and $w_{l}$ are weighting factors with values 0.75 and 0.25 respectively. These values were defined to represent the confident impact of the discrimination power of $\beta$ and $\sigma$ by analysis of their distribution values based on 6291 and 847 candidate regions of fire and non-fire regions. The threshold of 0.275 in Eq. (12) was also determined by statistical analysis of the mean values of the both distributions.

\section{Results and Conclusions}

\subsection{Experimental Results}

The method was tested in thermal videos with a resolution of 320x240 at 15 FPS acquired with a camera Fluke Ti45 working in the bands $8 \mu \mathrm{m}$ to $14 \mu \mathrm{m}$. The video set includes different types of situations in order to test the robustness of the method. Besides, a set of Internet videos acquired with a moving camera, low contrast and multiple fire regions were also included. Table 1 shows the information of the video data set. The complete data set and obtained results are available in http://dspvisionlab.itch.edu.mx/fjperez.

Table 1. Video data set

\begin{tabular}{llll}
\hline Video & Frames & Description & Camera \\
\hline NoFire 1 & 230 & Two walking people in a room & Static \\
NoFire 2 & 1692 & Controlled fire, lighter & Static \\
NoFire 3 & 815 & Pencil type soldering tin & Static \\
NoFire 4 & 182 & Walking person in a room & Static \\
\hline Fire 1 & 515 & Fire with Blue-Red palette & Static \\
Fire 2 & 286 & Fire & Moving \\
Fire 3 & 740 & Fire close to a person & Static \\
Fire 4 & 1081 & Fire with Blue-Red palette & Static \\
Fire 5 & 1551 & Firefighter controlling an indoor fire & Moving \\
Fire 6 & 742 & Fire video acquired from a helicopter & Moving \\
Fire 7 & 596 & Interior fire and explosion & Static \\
Fire 8 & 1216 & House on fire, part 1 & Moving \\
Fire 9 & 1185 & House on fire, part 2 & Moving \\
\hline
\end{tabular}


Figure 6 shows an example of a non-fire high temperature and fire regions processing including their features values. These values are consistent with the information aforementioned as well as the justification of the weighting factors.
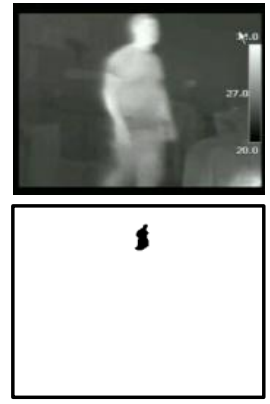

$\beta=0.156, \sigma=0.547, \gamma=0.254$
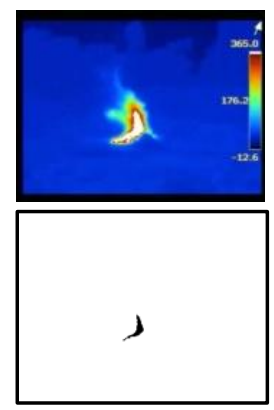

$\beta=0.254, \sigma=0.663, \gamma=0.356$

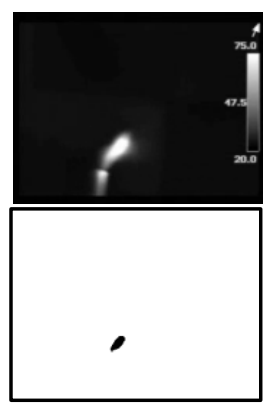

$\beta=0.025, \sigma=0.806, \gamma=0.220$
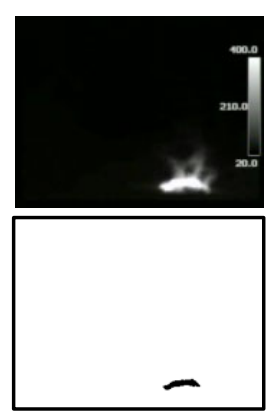

$\beta=0.439, \sigma=0.858, \gamma=0.544$
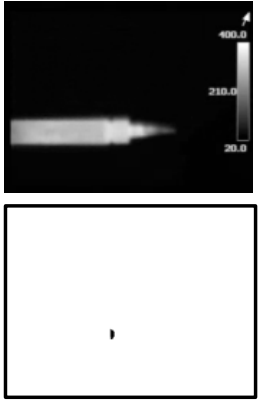

$\beta=0.018, \sigma=1.0, \gamma=0.263$ a)
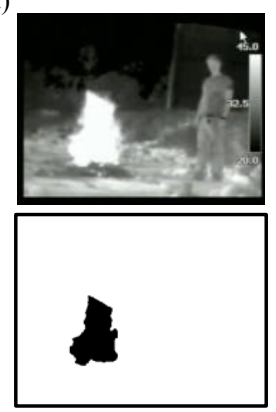

b)
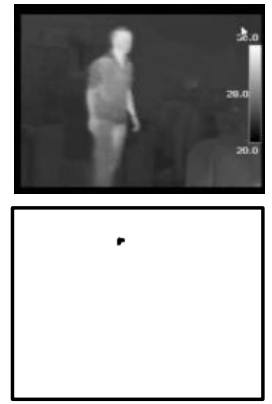

$\beta=0.009, \sigma=0.5, \gamma=0.1318$
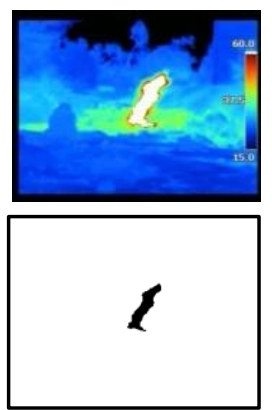

$\beta=0.570, \sigma=0.729, \gamma=0.625$

Fig. 6. a) Non-fire high temperature and, b) controlled fire regions with features values

On the other hand, Figure 7 shows cases of the Internet videos. These examples show the proposed method robustness under extreme conditions, low contrast, multiple fire regions and moving camera. These conditions make to fail other documented methods because they are based on fixed position pixel and temporal information.

\subsection{Performance Metrics}

The performance of the proposed method is presented in Table 2 and 3. A comparison with other methods was not directly achieved because data used by other methods was not available. The information provided is; number of processed frames, frames with fire, number of frames with fire detected, false positives and the percentages of hits, miss, and false positives. 
Results in Table 2 indicate that for non-fire videos the method performs well with an average of $4.99 \%$ of false positives. In regards fire detection Table 3 shows that the average percentage of hits is $75.06 \%$. Video 5 presents a high false positive rate because the fire region is very hard to define even by a person. The average performance is acceptable compared with other works [1][3] that report $66.4 \%$, $86.1 \%$, for true positives, $4.9 \%, 0.4 \%$ false positives, and $23.7 \%$ and $13.2 \%$ in missing rate. These methods do not consider the moving camera case or the multiregion fire situation. They work on the visible spectrum and do not use the same set of videos used in this work.

In conclusion, we can say that the proposed method has acceptable results in most of the tested situations and also compared with other methods based on color and temporal information which present a high false alarm rate. Also, the method shows robustness in moving camera videos which is not supported by methods based on temporal information. The current processing speed is 10.7 fps running in Matlab, therefore the method is guaranteed to run in real time.

For future work, we are currently developing a more sophisticated classification scheme based on Fuzzy Logic using the same features presented in this paper.
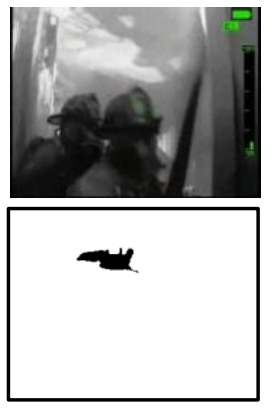

$\beta=0.457, \sigma=0.307, \gamma=0.419$
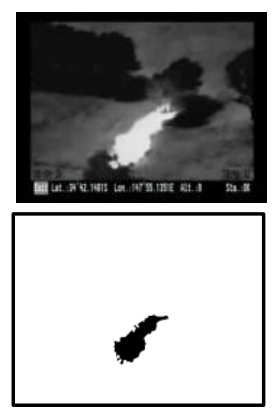

$\beta=0.509, \sigma=0.021, \gamma=0.386$
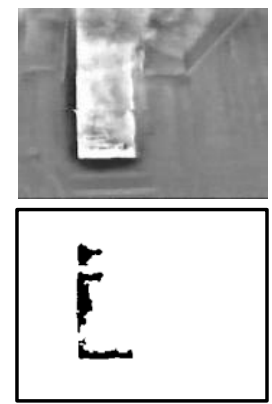

$\beta=[1,0.13,0.68], \sigma=[0.62,0.57,0.78]$, $\gamma=[0.92,0.24,0.707]$
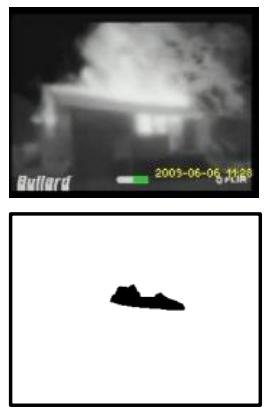

$\beta=0.707, \sigma=0.528, \gamma=0.662$

Fig. 7. Examples of extreme fire conditions processing and their features values

Table 2. Non-fire cases performance

\begin{tabular}{llccl}
\hline Video & \multicolumn{3}{c}{ Frames } & \%False positives \\
& Processed & Fire & False Positives \\
\hline NoFire 1 & 115 & 0 & 15 & $13.04 \%$ \\
NoFire 2 & 846 & 0 & 15 & $1.77 \%$ \\
NoFire 3 & 408 & 0 & 21 & $5.15 \%$ \\
NoFire 4 & 91 & 0 & 0 & $0.00 \%$ \\
Average & & & & $\mathbf{4 . 9 9 \%}$ \\
\hline
\end{tabular}


Table 3. Fire cases performance

\begin{tabular}{lrrrrrrr}
\hline Video & \multicolumn{2}{c}{ Frames } & & & \\
& Processed & Fire & Hits & $\begin{array}{l}\text { False } \\
\text { Positives }\end{array}$ & Hit\% & Miss\% & \multicolumn{1}{l}{$\begin{array}{l}\text { False } \\
\text { Positives\% }\end{array}$} \\
\hline Fire 1 & 257 & 209 & 144 & 0 & $68.90 \%$ & $31.10 \%$ & $0.00 \%$ \\
Fire 2 & 143 & 138 & 107 & 0 & $77.54 \%$ & $22.46 \%$ & $0.00 \%$ \\
Fire 3 & 370 & 218 & 180 & 3 & $82.57 \%$ & $17.43 \%$ & $1.97 \%$ \\
Fire 4 & 540 & 442 & 366 & 2 & $82.81 \%$ & $17.19 \%$ & $2.04 \%$ \\
Fire 5 & 775 & 630 & 390 & 89 & $61.90 \%$ & $38.10 \%$ & $61.38 \%$ \\
Fire 6 & 371 & 154 & 92 & 0 & $59.74 \%$ & $40.26 \%$ & $0.00 \%$ \\
Fire 7 & 298 & 296 & 293 & 0 & $98.99 \%$ & $1.01 \%$ & $0.00 \%$ \\
Fire 8 & 608 & 588 & 370 & 0 & $62.93 \%$ & $37.07 \%$ & $0.00 \%$ \\
Fire 9 & 592 & 590 & 473 & 0 & $80.17 \%$ & $19.83 \%$ & $0.00 \%$ \\
Average & & & & & $\mathbf{7 5 . 0 6 \%}$ & $\mathbf{2 4 . 9 4 \%}$ & $\mathbf{7 . 2 7 \%}$ \\
\hline
\end{tabular}

Acknowledgements. The authors thanks to Fondo Mixto de Fomento a la Investigación Científica y Tecnológica CONACYT-Gobierno del Estado de Chihuahua, by the support of this research under grant CHIH-2009-C02-125358. Special thanks to the SOFI de Chihuahua by providing the thermal equipment used in this research.

\section{References}

1. Toreyin, B.U., Dedeoglu, Y., Gudukbay, U., Cetin, A.E.: Computer Vision Based Method for Real-time Fire and Flame Detection. Pattern Recognition Letters 27, 49-58 (2006)

2. Phillips III, W., Shah, M., Lobo, N.V.: Flame Recognition in Video. Pattern Recogn. Letters 231(3), 319-327 (2002)

3. Ko, B.C., Cheong, K.H., Nam, J.Y.: Fire Detection Based on Vision Sensor and Support Vector Machines. Fire Safety Journal 44, 322-329 (2009)

4. Marbach, G., Loepfe, M., Brupbacher, T.: An Image Processing Technique for Fire Detection in Video Images. Fire Safety Journal 41, 285-289 (2006)

5. Uğur, B., Gökberk, R., Dedeoğlu, Y., Enis, A.: Fire Detection in Infrared Video Using Wavelet Analysis. Optical Engineering 46, 067204 (2007)

6. Kamgar-Parsi, B.: Improved image thresholding for object extraction in IR images. IEEE International Conference on Image Processing 1, 758-761 (2001)

7. Heriansyah, R., Abu-Bakar, S.A.R.: Defect detection in thermal image for nondestructive evaluation of petrochemical equipments. In: NDT \& E International, vol. 42(8), pp. 729-774. Elsevier, Amsterdam (2009)

8. Chacon, M.I.: Digital Image Processing (in spanish). Editorial Trillas (2007)

9. Gonzalez, R.C., Woods, R.E.: Digital Image Processing, 2nd edn., pp. 648-649. PrenticeHall, Englewood Cliffs (2002) 